\title{
The radiologist's role in lung cancer screening
}

\author{
Annemiek Snoeckx ${ }^{1}$, Caro Franck ${ }^{1}$, Mario Silva $^{2}$, Mathias Prokop $^{3}$, Cornelia Schaefer-Prokop ${ }^{4}$, \\ Marie-Pierre Revel ${ }^{5}$
}

${ }^{1}$ Department of Radiology, Antwerp University Hospital and University of Antwerp, Edegem, Belgium; ${ }^{2}$ Scienze Radiologiche, Department of Medicine and Surgery (DiMeC), University of Parma, Parma, Italy; ${ }^{3}$ Department of Medical Imaging, Radboud University Medical Center, Nijmegen, The Netherlands; ${ }^{4}$ Department of Radiology, Meander Medical Center, Amersfoort, The Netherlands; ${ }^{5}$ Department of Radiology, Cochin Hospital, APHP Centre, Université de Paris, Paris, France

Contributions: (I) Conception and design: All authors; (II) Administrative support: None; (III) Provision of study materials or patients: None; (IV) Collection and assembly of data: None; (V) Data analysis and interpretation: None; (VI) Manuscript writing: All authors; (VII) Final approval of manuscript: All authors.

Correspondence to: Annemiek Snoeckx, MD, PhD. Department of Radiology, Antwerp University Hospital, Wilrijkstraat 10, 2650 Edegem, Belgium. Email: Annemiek.snoeckx@uza.be.

\begin{abstract}
Lung cancer is still the deadliest cancer in men and women worldwide. This high mortality is related to diagnosis in advanced stages, when curative treatment is no longer an option. Large randomized controlled trials have shown that lung cancer screening (LCS) with low-dose computed tomography (CT) can detect lung cancers at earlier stages and reduce lung cancer-specific mortality. The recent publication of the significant reduction of cancer-related mortality by $26 \%$ in the Dutch-Belgian NELSON LCS trial has increased the likelihood that implementation of LCS in Europe will move forward. Radiologists are important stakeholders in numerous aspects of the LCS pathway. Their role goes beyond nodule detection and nodule management. Being part of a multidisciplinary team, radiologists are key players in numerous aspects of implementation of a high quality LCS program. In this non-systematic review we discuss the multifaceted role of radiologists in LCS.
\end{abstract}

Keywords: Lung cancer screening; imaging; radiologist; radiology; artificial intelligence (AI)

Submitted Aug 08, 2020. Accepted for publication Sep 24, 2020.

doi: 10.21037/tlcr-20-924

View this article at: http://dx.doi.org/10.21037/tlcr-20-924

\section{Introduction}

The high mortality of lung cancer in men and women worldwide is related to the fact that the diagnosis is most often made only after occurrence of symptoms, resulting in an advanced tumor stage, for which curative treatment is no longer possible $(1,2)$. The recently introduced immunotherapies for advanced lung cancer have improved short term survival, but at substantial cost, and without changing long-term lung cancer-specific mortality. In 2011, the National Lung Screening Trial (NLST) showed that participants who received low-dose computed tomography (LDCT) scans had a $20 \%$ lower risk of dying of lung cancer compared to participants who were screened with chest radiography (3). Two years later, the US Preventive
Services Task Force recommended lung cancer screening (LCS) with LDCT in smokers with a 30 -pack-year smoking history who currently smoke or have quit within the past 15 years (4). In 2015, LDCT was reimbursed by Medicare and Medicaid Services, with implementation of LCS throughout the US (5). Final data of the randomized Dutch-Belgian NELSON trial (Nederlands-Leuvens Longkanker Screenings Onderzoek) showed a lung cancerspecific mortality reduction of $24 \%$ in men and $33 \%$ in women at 10-year follow-up (6). Two other European studies confirmed the benefit of LCS, by showing a 39\% reduced risk of lung cancer mortality after 10 years in the Italian MILD trial (7). The German LUSI trial found a non-significant lung cancer-specific mortality reduction of $6 \%$ in men but a significant reduction in lung cancer- 
Table 1 The multifaceted role of radiologists in lung cancer screening

Quality assurance and continuous updating of local technical standards

Low-dose CT protocols

Conformance of software for computer-aided detection (CAD) and/or diagnosis (CADx) (e.g., make sure that volumetry conforms with reference standard

Overseeing day-to-day program functions

Pivotal role in the workflow of lung cancer screening program

Reading the scans and skilled management of findings, including extra-modular findings (e.g., coronary calcification, interstitial lung abnormalities). Of note, emphasis is placed on preferring conservative management of findings with relatively low clinical impact (e.g., control overdiagnosis)

Accurate communication of results through structured reporting

Active role in multidisciplinary lung cancer (screening) board

Monitoring performance standards (e.g., periodical analysis of rate of false negatives and false positives)

Dissemination and cultural exchange

Educating the public on imaging-based cancer screening

Participation in National-International quality assurance boards

Investigation, evaluation and implementation of artificial intelligence into different aspects of the lung cancer screening process

Collaboration and participation in research

specific mortality of $69 \%$ in women after an average of 8.8 years of follow-up (8).

In 2017, a European Position Statement on LCS set recommendations to guide the implementation of LCS in Europe and urged member states to take rapid action (9). Recently, the European Respiratory Society and European Society of Radiology published a statement paper on LCS, reviewing current practice, addressing unresolved issues and calling to convince policymakers across the European Union to start implementation of high-quality LCS programs in order to prevent unsupervised opportunistic screening $(10,11)$. European guidelines exist for populationbased screening of breast cancer, cervical cancer and colon cancer, but upon today there are no European guidelines for LCS. Hope is that in the light of the recent NELSON results, implementation of LCS in Europe will move forward.

Implementation will be different in the various European countries, depending on the National Health Service model and national organization of population-based screening and will be adopted to local health care facilities. Nevertheless, there will also be a common ground on how LCS will be organized. Ensuring high-quality LCS programs will require close collaboration with many stakeholders, in which radiologists play a crucial role. In order to set quality guidelines and emphasize expertise and quality assurance, the European Society of Thoracic Imaging has developed a Lung Cancer Certification Project (12). The objective is to standardize training and expertise quality across Europe and to ensure that LCS is practiced at a similar quality level to the trials that have proven the value of screening.

This manuscript discusses the multifaceted role of radiologists in LCS (Table 1).

\section{Low-dose CT}

In an effective screening program for an asymptomatic population, the potential benefit for early cancer detection must outweigh harmful effects to the individual and population as a whole. In LCS, CT radiation exposure is one of the harms. Although the risk of radiation-induced cancer is low compared with the benefits of a LCS program, this risk rises proportionally with the radiation dose used. Radiation dose levels of CT examinations for LCS can be kept very low, thanks to the inherent high contrast level between lung parenchyma and nodules. Specific low-dose protocols, consistent with the balance of dose reduction and necessary image quality, have to be put in place in a LCS program. Development and optimization of these LDCT protocols should be established in close collaboration 
by thoracic radiologists and medical physicists. Despite guidelines from the American College of Radiology (ACR) on recommended CT radiation dose levels (13), a cohort study of 12,529 participants at 72 institutions in the US found a wide variation in the distribution of LCS CT doses across facilities (14). According to the authors, $21 \%$ of the institutions had median volume computed tomography dose index $\left(\mathrm{CTDI}_{\mathrm{vol}}\right)$ above ACR benchmarks ( $>3 \mathrm{mGy}$ ). Institutions where the lead radiologist and/or medical physicist established LDCT protocols had lower doses, whereas institutions where radiologists and physicists did not collaborate had higher doses.

As described in the European Federation of Organisations for Medical Physics (EFOMP) policy statement, a medical physicist has the knowledge, training and experience to give advice on matters related to medical radiation exposure (15). Apart from acceptance testing and quality assurance of the CT equipment used in the screening program, a medical physicist can train radiologists and other staff members in relevant aspects of radiation protection. Having an in-house medical physicist who is actively involved in CT protocol optimization can help radiologists to better manage LCS participant doses. In addition, medical physicists are responsible for comparing radiation doses against national reference levels, so that hospitals with in-house physicists will better maintain appropriate radiation dose levels.

Initial Medicare recommendations used effective dose for LDCT guidelines. Because of inconsistencies in the calculation of effective dose, the American Association of Physicists in Medicine (AAPM) and ACR argued for the use of $\mathrm{CTDI}_{\mathrm{vol}}$, a dose metric which is displayed on the scanner. Current recommendations from the European Society of Thoracic Imaging (16) recommend a CTDI ${ }_{\mathrm{vol}}$ of 0.4-1.6 mGy, depending on the weight of the patient. Scanning parameters should be set so that scans can be performed in single-breath-hold ( $\leq 10 \mathrm{~s})$. The use of automatic exposure control, such as adjustment of the tube current according to the size and the anatomy of the patient, is mandatory. Since LCS is performed without intravenous (IV) contrast, the contrast between noncalcified nodules and background does not increase with low $\mathrm{kV}$ scanning, while image noise does increase. Low-kV scanning or automatic adjustment of tube potential is therefore not recommended. Thin slices $(\leq 1.0 \mathrm{~mm})$ should be reconstructed to ensure the detection of small lesions, and reconstruction increments should be even smaller $(\leq 0.7 \mathrm{~mm})$ to optimize quantitative measurement of nodules. Filtered back projection should no longer be used for reconstruction of screening scans. Instead, modern techniques such as iterative and deep learning-based image reconstruction techniques are mandatory. Thoracic radiologists and medical physicists are in the frontline to continuously follow the technical evolution of CT in order to monitor and optimize both quality and radiation exposure of LDCT.

CT-examinations in general are being performed in a hospital- or clinic-based setting. From a participant perspective, different barriers to LCS exist, of which access is one of them. A survey of non-participants in the UK Lung Cancer Screening (UKLS) showed that the most common reason (reported by $18 \%$ ) for declining participation in LCS was travel-related difficulties to overcome the distance between home and the screening site (17). A mobile LDCT unit, analogous to mobile mammography, offers improved access, for example in car parks or public spaces (18). This was part of the success of the Manchester study (19), in which ever-smokers were invited to "lung health checks" next to local shopping centers, with immediate access to LDCT for those at high risk. However, one has to make sure that these mobile scanners fulfil the quality criteria described above. Raghavan et al. investigated the effect of a mobile screening unit for LCS in the US. Their results clearly show that a mobile unit allows effective LCS for underserved populations (20). In Europe, the United Kingdom is the country that has the most experience with use of mobile CT trucks, with different LCS projects across the country (21).

\section{Reading}

\section{Role of computer-aided detection (CAD)}

Radiologists play a crucial role in one of the key elements of LCS. Analogous to breast cancer screening, most screening trials implemented double reading, at least at the start of the study (22-25). NLST was an exception, with single reads per examination by experts who went through an approval process prior to the study (3). Sensitivity of detection of small pulmonary nodules is a challenging task, depending on reader, nodule size and morphology, associated parenchymal abnormalities or underlying disease and technical parameters of the CT scan. Intra- and interobserver agreement in detecting pulmonary nodules in LDCT is frequently limited (26).

To improve lesions detection, CAD algorithms are 
increasingly being used. CAD systems have proven to outperform radiologists as second reader (27-29). There is a multitude of studies evaluating the performance of CAD under various conditions. It has to be noted that CAD systems vary quite remarkable in performance and that the current development speed of computerized tools to support reading and classifying lung lesions is so fast that any conclusions about application of CAD should always be drawn in the appropriate context.

Nevertheless, Christe et al. showed that the human aspect remains important. In their study, human readers with CAD assistance had consistently higher sensitivity $(97-99 \%)$ than any combination of two CAD systems (85-88\%) (30). Liang et al. (31) showed that various CAD systems were able to detect $56-70 \%$ of missed lung cancers. Conversely, CAD missed $21 \%$ of cancers that were identified by radiologists. Silva et al. compared the performance of CAD and visual reading for detection of subsolid nodules. CAD had higher sensitivity than visual reading $(88.4 \%$ versus $34.2 \%)$. Nonetheless, CAD detected only $66 \%$ of the subsolid nodules seen by visual detection (32). Results thus far suggest that CAD and radiologists have a complimentary value and that the combination of CAD and visual reading will lead to an optimal detection performance, not only for solid nodules but especially for subsolid nodules. Radiologists expertise will outperform CAD systems in participants with underlying lung disease or in cases where early lung cancers don't present as pulmonary nodules. Analysis of interval and postscreen carcinomas in the NELSON trial showed that of the missed carcinomas were related to endobronchial location, lesions attached to a bulla or subpleural location (33). Lung cancers associated with cystic airspaces is an entity that gained increasing recognition over the past years and is hard to pick up by current CAD systems due to the different morphology compared to typical pulmonary nodules $(34,35)$.

Presently, most CAD systems for LCS serve as a concurrent reader, meaning the CAD reading should be associated with human reading. CAD performance will however improve with deep learning-based approaches. The deep learning-based model proposed by Ardila et al. outperformed all six radiologists in this study for predicting the risk of lung cancer on low-dose chest CT when prior CT imaging was not available, whereas the model performance was on-par with the same radiologists if they had access to prior CT examinations (36). With the (r) evolution of artificial intelligence (AI), the performance of CAD will improve, and its role may shift from second reader to first reader in the future.

\section{Volumetric versus diameter measurements}

Once detected, radiological assessment of pulmonary nodules is primarily based on size and morphology. Nodule evaluation in the NLST was performed by manual diameter measurement (3). Manual measurement of lesion diameter is prone to a large amount of variability (37-39). Numerous European trials, including NELSON $(25,40)$, Danish LCS trial (41), UKLS trial (42) and Multicentric Italian Lung Detection (MILD) trial (7) used volumetric evaluation of pulmonary nodules for optimal characterization of volume increase and, therefore, early signs of growth. Volumetric measurements are semi-automatically performed by certified nodule evaluation software. Volumetry provides nodule segmentation in all three dimensions, even for complex nodules. As a consequence, volumetric measurements show a better intra-observer and inter-observer variability than diameter measurements, notably for nodules without surface contact with solid structures (43). Volumetric measurements can vary between different volumetry software packages and can have an impact on nodule management (44-47). Using identical software packages and even software versions for follow-up is therefore mandatory (48). Radiologists involved in LCS should be aware of the benefits and drawbacks of volumetry packages, advantages and limitations of nodule volumetry. They should be aware of the impact of acquisition, reconstruction, patient and reader factors of volumetry reliability. They should also be aware of the fact that it is important that the software package used in their screening program is comparable to those used to set guidelines (46). In Europe, volumetry has been recommended as the preferred method of nodule size measurement in LCS $(9-11,16)$.

\section{Nodule classification beyond size}

Whereas size is the most important factor for nodule management, nodule morphology is nevertheless important $(49,50)$. Pulmonary nodule management is a challenging task that should be in hands of experienced radiologists in order to optimize early detection of cancer and minimize any potential harm (unnecessary workup and invasive interventions). Both LU-RADS (51), Lung-RADS (52) and the Brock model (53) are nodule management systems for LCS that use nodule morphology, assessed by the 
radiologist, as a decision criterion. Lung-RADS, however, is the only system that uses a "gestalt" approach in which the radiologist can upgrade the risk profile of a nodule based on integral assessment of nodule morphology. Chung et al. showed substantial added value of radiologists upgrading subsolid lesions to a higher malignancy risk category (LungRADS category $4 \mathrm{X}$ over categories 3, 4A and 4B), with high malignancy rates in the hands of experienced radiologists (54). Deep learning based computerized assessment of nodule morphology is expected to substantially improve the malignancy risk assessment of nodules, most likely beyond the capacity of visual assessment alone $(55,56)$.

\section{Strategies to reduce costs}

Ritchie et al. investigated the role of a non-radiologist technician assisted by CAD for finding abnormal LDCT scans for subsequent review by a radiologist. Their results showed that this setup for reading LCS studies is a promising strategy for reducing workload of radiologists, improving speed, consistency and quality of scan interpretation and, last but certainly not least, to reduce costs (57). Nair et al. compared the performance of radiographers against that of radiologists for CT lung nodule detection in the UKLS pilot trial. Their overall results showed that radiographer performance was lower than that of experienced radiologists and a role as first reader would currently not be an option (58). In a follow-up study, the same authors investigated radiologists' performance reading CTs independently with radiographers as concurrent readers, showing that radiologists' sensitivity in lung nodule detection could be improved with this reading strategy.

Optimization of resources for LCS is also achievable by post-test stratification of lung cancer risk, with reduction of LCS intensity in cases in which this risk is low. This results in a reduction of per-subject number of CTs. Several authors reported (prospectively and retrospectively) that screening intervals of more than 1 year are feasible for screenees with minor CT findings who represent a large proportion of the screening population and for whom the mid-term risk of lung cancer is substantially lower than the mean risk (59-63). This option will be soon explored in the largest European randomized trial called 4InTheLungRun (H2020-SC1-BHC-2018-2020; grant848294; 2019).

\section{Reporting}

Accurate communication of results is essential for any screening program. Reporting in a "structured" way simplifies standardization of management and facilitates data collection and mining as well as monitoring of performance of different aspects of the LCS program. Strict algorithms will enhance quality of the LCS program. Standardized terminology and structure improve clarity and communication of findings for both participants and physicians involved in screening (64). Structured reporting is one of the recommendations of the ACR as part of accreditation of LCS programs. For this purpose, ACR has developed Lung-RADS, with specific assessment categories and management recommendations for screen-detected pulmonary nodules (65).

Times are changing and medicine is gently transitioning toward a more patient-centered approach. With the digital evolution, patients are having more and more access to their own medical data, including medical images. They are taking a more central role in their own care. In the setting of screening, shared or "informed" decisionmaking has become a critical part. Where patients in the past traditionally were informed of imaging results by their referring physician, nowadays they often have access to both images and medical reports. Radiology reports are a communication tool with referring physicians $(52,66)$ and are therefore not easy to understand for a patient. A dedicated participant-friendly LCS report, designed with feedback of participants and patient advocacy groups, may improve understanding of results. Good understanding of results might improve adherence to the screening program and follow-up recommendations. Vitzthum et al. stated that "radiologists bave an opportunity to empower patients and participate in the patient-centered care movement by providing patients with a better understanding of their medical condition and the possible steps in care moving forward through improved patient-centered reporting" (67).

\section{More than meets the eye}

LCS with LDCT primarily focuses on early detection of lung cancer by finding pulmonary nodules and assessing their risk of representing a cancer. Chest CTs display far more than only the lungs, offering the opportunity to asses more than only pulmonary nodules. The targeted population for LCS, being current or former smokers, is prone to more than only development of lung cancer. They are also at risk for premature death of chronic obstructive pulmonary disease (COPD) and heart disease. Coronary artery calcifications are a valuable biomarker to determine the risk of cardiovascular events. They can 
be detected and quantified on non-gated LDCTs for LCS (68). Data from the NELSON trial confirmed the previous observations on the strong predictive value of coronary artery calcifications beyond age, pack-years and smoking status (69). The association between outcomes and coronary artery calcification was studied by Watts et al. who investigated a group of 1,000 participants who died in NLST. An ordinal scoring system and visual score based on linear extent and thickness of calcification was used for this study. Results showed that the presence of any calcium was associated with a 3 -fold increase in cardiovascular deaths with an odds ratio of 3.4 (95\% CI: 2.0-5.6) and 3.3 (95\% CI: 2.0-5.6) for both scoring systems. Furthermore, an increasing risk was associated with an increasing score (70). LDCT also offers the opportunity for early detection of COPD, by quantifying emphysema, airway wall thickness and air trapping, with most experience been gained with emphysema quantification. There is indirect evidence to suggest that emphysema is a predictive factor for the development of lung cancer (71). Heuvelmans et al. propose different combinations of acquisition protocols for quantification of lung nodules, emphysema, air trapping and coronary artery calcification, also discussing pros and cons of each option (72). Targeting the "big-3" may not only decrease the disease burden and save more lives but may significantly improve cost-effectiveness of a future LCS program. Quantification of findings on LDCT may even target more than the "big-3", with the possibility of quantifying numerous aspects, including airways (73), vascular calcifications (74), bone density (75), breast density (76), and liver density (77). Pleural plaques are a common finding on chest CT studies and are related to asbestos exposure. Asbestos exposure is not only a risk factor for development of malignant pleural mesothelioma, but also for development of lung cancer. Silva et al. investigated the presence of pleural plaques in LCS participants and relationship with lung cancer and mortality. They showed that male screenees with pleural plaques showed a trend for higher risk of lung cancer and significant increase of lung cancer mortality compared to male screenees without pleural plaques with a hazard radio of 5.48 (95\% CI: 1.6118.70) for mortality of lung cancer in subjects with pleural plaques (78).

Quantitative imaging of all these entities beyond pulmonary nodules is eased by the recent increasing role of AI. Software development for quantification is taking huge steps forward. A number of imaging biomarkers are yet ready for use in daily practice. What will be far more challenging is how to deal with this huge amount of quantitative data and additional information and how to incorporate it in a screening program without increasing harm to participants. Currently, evidence of the benefit of screening in terms of health-related quality of life, morbidity and mortality is lacking for the majority of these imaging biomarkers. Randomized controlled trials with inclusion of validated, quantified imaging biomarkers for other diseases than lung cancer are needed for the future.

\section{Coming out of the dark}

Radiologists' task in LCS goes beyond the aspects happening in the radiology department, including setting the scan protocol, reading and reporting and overseeing of day-to-day program functions. Radiologists should have an active role in a multidisciplinary team which aims to minimize harm and to ensure that participants receive the most appropriate treatment. Close teamwork and effective communication with all stakeholders involved in LCS is essential. They should also be part of national quality assurance boards, set up by professional bodies to ensure adherence to technical standards.

Despite compelling evidence in support of LCS, uptake has not been a big success (79). Targeting the hard-to reach will be challenging. Where radiologists are typically invisible for patients $(80,81)$ a more visible role in educating the public on imaging-based cancer screening might be important in LCS. Radiologists have a substantial role in LCS and therefore definitely have added value regarding education on this topic. Rosenkrantz et al. showed a strong impact of teaching sessions in increasing participants' awareness and knowledge on imaging-based screening tests (82). Enhancing knowledge will have a positive impact on shared decision making, which is an essential part of LCS. Shareddecision making is a collaborative process between healthcare providers and participants allowing decisions to be made together, taking into account both benefits and harms (83). The decision to take part or to decline has to be in line with participants' individual values. Educating the public, will empower radiologists to positively impact targeting the hard-to-reach. The latter still being one of the biggest challenges in LCS.

With increasing emphasis on a patient-centered approach to radiology, radiologists will have growing responsibilities in communicating directly to patients. LCS programs might provide an opportunity to radiologists to meet, consult and educate participants. Radiologists are good 
resources for participants to discuss screening with LDCT, radiation risk and CT findings. The nature of this contact however strongly depends on preferences from radiologists, participants and referring physicians as well as practical concerns (84).

\section{Artificial intelligence}

AI is reshaping healthcare and the imaging world. With radiology being a discipline driven by digital data, radiologists are on the frontline of $\mathrm{AI}$ innovation in the medical sector. Radiologists are faced with ever-increasing volume of digital data. One of the logistical questions and hurdles on implementation of nationwide LCS programs is the capacity of radiologists and/or other trained individuals. CAD will be necessary to relief pressure on radiologists and radiology services. In contrast to humans, computers can handle high volumes and are consistent. AI algorithms will play a role in different tasks of the screening process. As previously discussed, for now the most appropriate role of CAD is that of a second reader, in order to increase sensitivity for nodule detection. To be of value, high sensitivity of CAD in terms of nodule detection is necessary, balanced with a low rate of false-positive findings. AI can also have a role in automatically categorizing screen detected pulmonary nodules (85). Volumetric assessment with comparison with the previous examination and calculation of volume doubling time is already in practice. $\mathrm{AI}$ algorithms for malignancy prediction will become more accurate in the future as more data of LCS programs will be available, but for the time being, radiologist can only validate detections by their visual confirmation, whereas validating malignancy prediction still relies on histology. Computer-aided diagnosis systems (CADx) may also reduce variability in assessing and reporting lung cancer risk between physicians (86). Baldwin et al. recently reported a convolutional neural network system capable of higher accuracy than the multidimensional risk model from Brock University (56). Numerous aspects however need to be investigated before integration of CADx in a clinical setting is possible. The role of AI in LCS goes beyond nodule detection, volumetric assessment and nodule characterization. Deep learning denoising algorithms have the potential to significantly reduce dose, without hampering image quality. Further research on role of these algorithms in LCS is however needed. Currently, the role of AI in participant selection and screening planning is less straightforward, but it is without doubt that if large amounts of data will be collected through implementation of screening in daily practice, that this field will also move forward.

Radiologists should be aware of not only the advantages, but also limitations of the AI algorithms that they use. AI and radiologists will need to closely work together, but the exact format of this collaboration still needs further investigation.

\section{Research opportunities}

Global implementation of LCS has just started. There are many unanswered questions and problems to be addressed. European implementation trials are needed, investigating tailored invitations for targeting the hard to reach, studies on screening intervals, integration of other imaging biomarkers, blood- or breath-based biomarkers for early lung cancer detection, smoking cessation, and quality assurance (87).

There is one big under-researched area in screening, however, namely how to identify those individuals at risk for lung cancer who are currently not covered by current LCS eligibility criteria. More than $50 \%$ of patients with lung cancer would not have fulfilled current criteria for LCS because they have never smoked or stopped smoking long ago $(88,89)$. Research is urgently needed on how to modify inclusion criteria intelligently to include those who might benefit most from early tumor detection because their risk of smoking-induced comorbidities is minimal. Conversely, research is needed on whom not to screen because their risk of dying from their comorbidities is substantially higher than their risk of dying from lung cancer. In those individuals, the burden of screening, especially false positives or slow-growing tumors causing over-diagnosis and over-treatment, may outweigh the benefits.

Continuous research and audits are essential to ensure persistent high-quality performance of LCS programs. Large-scale implementation of LCS will create large amounts of data. Data that need to be reported and stored in national registries. Collection on an international level will create the opportunity to accelerate the speed of innovation. In an attempt to gather metadata on an international level, the International Association for the Study of Lung Cancer (IASLC) is developing the Early Lung Imaging Confederation (ELIC). ELIC is a global computing environment with a hub-and-spoke architecture: images and metadata are retained at the local site, consistent with local governing data-sharing regulations. Data can be made 
accessible with local site permission and only the resulting analysis will leave an individual spoke (90). Recent laws on GDPR (European General Data Protection Regulation) will make sharing anonymized CT-images and data extremely challenging and numerous issues on this topic need to be addressed. Challenges that need to be tackled, because creation of a huge cloud-based image collection and collection of "big data" provides enormous potential to accelerate research on early lung cancer detection.

\section{Conclusions}

The radiologists' role in LCS goes beyond reading chest CTs and evaluating pulmonary nodules. Being part of a multidisciplinary team, radiologists are key players in numerous aspects of implementation of a high quality LCS program. The role of AI in LCS still needs to be unraveled, but it is without doubt that AI will put its mark on how LCS will be organized in the future. LCS in Europe is at the crossroad from trial to practice; this creates opportunities for cutting-edge research projects to answer the remaining questions and optimize LCS.

\section{Acknowledgments}

Funding: None.

\section{Footnote}

Provenance and Peer Review: This article was commissioned by the Editorial Office, Translational Lung Cancer Research for the series "Lung cancer screening". The article has undergone external peer review.

Conflicts of Interest: All authors have completed the ICMJE uniform disclosure form (available at http://dx.doi. org/10.21037/tlcr-20-924). The series "Lung cancer screening" was commissioned by the editorial office without any funding or sponsorship. AS served as the unpaid Guest Editor of the series. MP reports personal fees from Bracco, personal fees from Bayer, personal fees from Canon Medical Systems, personal fees from Siemens Healthineers, grants from Canon Medical Systems, grants from Siemens Healthineers, outside the submitted work. The authors have no other conflicts of interest to declare.

Ethical Statement: The authors are accountable for all aspects of the work in ensuring that questions related to the accuracy or integrity of any part of the work are appropriately investigated and resolved.

Open Access Statement: This is an Open Access article distributed in accordance with the Creative Commons Attribution-NonCommercial-NoDerivs 4.0 International License (CC BY-NC-ND 4.0), which permits the noncommercial replication and distribution of the article with the strict proviso that no changes or edits are made and the original work is properly cited (including links to both the formal publication through the relevant DOI and the license). See: https://creativecommons.org/licenses/by-nc-nd/4.0/.

\section{References}

1 Bray F, Ferlay J, Soerjomataram I, et al. Global cancer statistics 2018: GLOBOCAN estimates of incidence and mortality worldwide for 36 cancers in 185 countries. CA Cancer J Clin 2018;68:394-424.

2. de Groot PM, Wu CC, Carter BW, et al. The epidemiology of lung cancer. Transl Lung Cancer Res 2018;7:220-33.

3. Aberle DR, Adams AM, Berg CD, et al. Reduced lungcancer mortality with low-dose computed tomographic screening. N Engl J Med 2011;365:395-409.

4. Moyer VA; U.S. Preventive Services Task Force. Screening for lung cancer: U.S. Preventive Services Task Force recommendation statement. Ann Intern Med 2014;160:330-8.

5. Centers for Medicare \& Medicaid Services. Decision Memo for Screening for Lung Cancer with Low Dose Computed Tomography (LDCT) (CAG-00439N) [Internet]. 2015 [cited 2015 Jun 29]. Available online: https://www.cms.gov/medicare-coverage-database/details/ nca-decision-memo.aspx? NCAId $=274$

6. de Koning HJ, van der Aalst CM, de Jong PA, et al. Reduced Lung-Cancer Mortality with Volume CT Screening in a Randomized Trial. N Engl J Med 2020;382:503-13.

7. Pastorino U, Silva M, Sestini S, et al. Prolonged lung cancer screening reduced 10-year mortality in the MILD trial: new confirmation of lung cancer screening efficacy. Ann Oncol 2019;30:1162-9.

8. Becker N, Motsch E, Trotter A, et al. Lung cancer mortality reduction by LDCT screening-Results from the randomized German LUSI trial. Int J Cancer 2020;146:1503-13.

9. Oudkerk M, Devaraj A, Vliegenthart R, et al. European 
position statement on lung cancer screening. Lancet Oncol 2017;18:e754-66.

10. Kauczor HU, Baird AM, Blum TG, et al. ESR/ERS statement paper on lung cancer screening. Eur Radiol 2020;30:3277-94.

11. Kauczor HU, Baird AM, Blum TG, et al. ESR/ERS statement paper on lung cancer screening. Eur Respir J 2020;55:1900506.

12. European Society of Thoracic Imaging. ESTI Lung Cancer Screening Certification Project [Internet]. 2019 [cited 2020 Mar 12]. Available online: https://www.myesti. org/lungcancerscreeningcertificationproject/

13. Kazerooni EA, Austin JH, Black WC, et al. ACR-STR practice parameter for the performance and reporting of lung cancer screening thoracic computed tomography (CT): 2014 (Resolution 4). J Thorac Imaging 2014;29:310-6.

14. Demb J, Chu P, Yu S, et al. Analysis of Computed Tomography Radiation Doses Used for Lung Cancer Screening Scans. JAMA Intern Med 2019;179:1650-7.

15. Caruana CJ, Tsapaki V, Damilakis J, et al. EFOMP policy statement 16: The role and competences of medical physicists and medical physics experts under 2013/59/ EURATOM. Phys Med 2018;48:162-8.

16. European Society of Thoracic Imaging. ESTI LCS technical standards [Internet]. 2019 [cited 2020 Mar 12]. Available online: https://www.myesti.org/lungcancerscreen ingcertificationproject/

17. Ali N, Lifford KJ, Carter B, et al. Barriers to uptake among high-risk individuals declining participation in lung cancer screening: a mixed methods analysis of the UK Lung Cancer Screening (UKLS) trial. BMJ Open 2015;5:e008254.

18. Quaife SL, Marlow LAV, McEwen A, et al. Attitudes towards lung cancer screening in socioeconomically deprived and heavy smoking communities: informing screening communication. Health Expect 2017;20:563-73.

19. Crosbie PA, Balata H, Evison M, et al. Implementing lung cancer screening: baseline results from a communitybased 'Lung Health Check' pilot in deprived areas of Manchester. Thorax 2019;74:405-9.

20. Raghavan D, Wheeler M, Doege D, et al. Initial Results from Mobile Low-Dose Computerized Tomographic Lung Cancer Screening Unit: Improved Outcomes for Underserved Populations. Oncologist 2020;25:e777-81.

21. National Health Service. NHS to rollout lung cancer scanning trucks across the country [Internet]. 2019 [cited 2020 Mar 12]. Available online: https://www.england.nhs. uk/2019/02/lung-trucks/

22. Baldwin DR, Duffy SW, Wald NJ, et al. UK Lung Screen (UKLS) nodule management protocol: modelling of a single screen randomised controlled trial of low-dose CT screening for lung cancer. Thorax 2011;66:308-13.

23. Infante $M$, Lutman FR, Cavuto $S$, et al. Lung cancer screening with spiral CT. Lung Cancer 2008;59:355-63.

24. Pedersen JH, Ashraf H, Dirksen A, et al. The Danish Randomized Lung Cancer CT Screening Trial-Overall Design and Results of the Prevalence Round. J Thorac Oncol 2009;4:608-14.

25. Xu DM, Gietema H, de Koning H, et al. Nodule management protocol of the NELSON randomised lung cancer screening trial. Lung Cancer 2006;54:177-84.

26. Leader JK, Warfel TE, Fuhrman CR, et al. Pulmonary Nodule Detection with Low-Dose CT of the Lung: Agreement Among Radiologists. AJR Am J Roentgenol 2005;185:973-8.

27. Rubin GD, Lyo JK, Paik DS, et al. Pulmonary nodules on multi-detector row CT scans: performance comparison of radiologists and computer-aided detection. Radiology 2005;234:274-83.

28. Teague SD, Trilikis G, Dharaiya E. Lung nodule computer-aided detection as a second reader: influence on radiology residents. J Comput Assist Tomogr 2010;34:35-9.

29. Zhao Y, de Bock GH, Vliegenthart R, et al. Performance of computer-aided detection of pulmonary nodules in low-dose CT: comparison with double reading by nodule volume. Eur Radiol 2012;22:2076-84.

30. Christe A, Leidolt L, Huber A, et al. Lung cancer screening with CT: Evaluation of radiologists and different computer assisted detection software (CAD) as first and second readers for lung nodule detection at different dose levels. Eur J Radiol 2013;82:e873-8.

31. Liang M, Tang W, Xu DM, et al. Low-Dose CT Screening for Lung Cancer: Computer-aided Detection of Missed Lung Cancers. Radiology 2016;281:279-88.

32. Silva M, Schaefer-Prokop CM, Jacobs C, et al. Detection of Subsolid Nodules in Lung Cancer Screening. Invest Radiol 2018;53:441-9.

33. Scholten ET, Horeweg N, de Koning HJ, et al. Computed tomographic characteristics of interval and post screen carcinomas in lung cancer screening. Eur Radiol 2015;25:81-8.

34. Snoeckx A, Dendooven A, Carp L, et al. Wolf in Sheep's Clothing: Primary Lung Cancer Mimicking Benign Entities. Lung Cancer 2017;112:109-17. 
35. Snoeckx A, Reyntiens P, Carp L, et al. Diagnostic and clinical features of lung cancer associated with cystic airspaces. J Thorac Dis 2019;11:987-1004.

36. Ardila D, Kiraly AP, Bharadwaj S, et al. End-to-end lung cancer screening with three-dimensional deep learning on low-dose chest computed tomography. Nat Med 2019;25:954-61.

37. Erasmus JJ, Gladish GW, Broemeling L, et al. Interobserver and Intraobserver Variability in Measurement of Non-Small-Cell Carcinoma Lung Lesions: Implications for Assessment of Tumor Response. J Clin Oncol 2003;21:2574-82.

38. Gierada DS, Pilgram TK, Ford M, et al. Lung cancer: interobserver agreement on interpretation of pulmonary findings at low-dose CT screening. Radiology 2008;246:265-72.

39. Singh S, Pinsky P, Fineberg NS, et al. Evaluation of reader variability in the interpretation of follow-up CT scans at lung cancer screening. Radiology 2011;259:263-70.

40. van Klaveren RJ, Oudkerk M, Prokop M, et al. Management of Lung Nodules Detected by Volume CT Scanning. N Engl J Med 2009;361:2221-9.

41. Saghir Z, Dirksen A, Ashraf H, et al. CT screening for lung cancer brings forward early disease. The randomised Danish Lung Cancer Screening Trial: status after five annual screening rounds with low-dose CT. Thorax 2012;67:296

42. Field JK, Duffy SW, Baldwin DR, et al. UK Lung Cancer RCT Pilot Screening Trial: baseline findings from the screening arm provide evidence for the potential implementation of lung cancer screening. Thorax 2016;71:161-70.

43. Devaraj A, van Ginneken B, Nair A, et al. Use of Volumetry for Lung Nodule Management: Theory and Practice. Radiology 2017;284:630-44.

44. de Hoop B, Gietema H, van Ginneken B, et al. A comparison of six software packages for evaluation of solid lung nodules using semi-automated volumetry: What is the minimum increase in size to detect growth in repeated CT examinations. Eur Radiol 2009;19:800-8.

45. Liang M, Yip R, Tang W, et al. Variation in Screening CTDetected Nodule Volumetry as a Function of Size. AJR Am J Roentgenol 2017;209:304-8.

46. Soo E, Edey AJ, Mak SM, et al. Impact of choice of volumetry software and nodule management guidelines on recall rates in lung cancer screening. Eur J Radiol 2019;120:108646.

47. Zhao YR, van Ooijen PM, Dorrius MD, et al. Comparison of three software systems for semi-automatic volumetry of pulmonary nodules on baseline and follow-up CT examinations. Acta Radiologica 2014;55:691-8.

48. Ashraf H, de Hoop B, Shaker SB, et al. Lung nodule volumetry: segmentation algorithms within the same software package cannot be used interchangeably. Eur Radiol 2010;20:1878-85.

49. Larici AR, Farchione A, Franchi P, et al. Lung nodules: size still matters. Eur Respir Rev 2017;26:170025.

50. Snoeckx A, Reyntiens P, Desbuquoit D, et al. Evaluation of the solitary pulmonary nodule: size matters, but do not ignore the power of morphology. Insights Imaging 2018;9:73-86.

51. Manos D, Seely JM, Taylor J, et al. The Lung Reporting and Data System (LU-RADS): a proposal for computed tomography screening. Can Assoc Radiol J 2014;65:121-34.

52. Lung RADS [Internet]. [cited 2020 Aug 3]. Available online: https://www.acr.org/Clinical-Resources/ Reporting-and-Data-Systems/Lung-Rads

53. McWilliams A, Tammemagi MC, Mayo JR, et al. Probability of cancer in pulmonary nodules detected on first screening CT. N Engl J Med 2013;369:910-9.

54. Chung K, Jacobs C, Scholten ET, et al. Lung-RADS Category 4X: Does It Improve Prediction of Malignancy in Subsolid Nodules? Radiology 2017;284:264-71.

55. van Riel SJ, Ciompi F, Wille MMW, et al. Malignancy risk estimation of pulmonary nodules in screening CTs: Comparison between a computer model and human observers. PLoS One 2017;12:e0185032.

56. Baldwin DR, Gustafson J, Pickup L, et al. External validation of a convolutional neural network artificial intelligence tool to predict malignancy in pulmonary nodules. Thorax 2020;75:306-12.

57. Ritchie AJ, Sanghera C, Jacobs C, et al. Computer Vision Tool and Technician as First Reader of Lung Cancer Screening CT Scans. J Thorac Oncol 2016;11:709-17.

58. Nair A, Gartland N, Barton B, et al. Comparing the performance of trained radiographers against experienced radiologists in the UK lung cancer screening (UKLS) trial. Br J Radiol 2016;89:20160301.

59. Patz EF, Greco E, Gatsonis C, et al. Lung cancer incidence and mortality in National Lung Screening Trial participants who underwent low-dose CT prevalence screening: a retrospective cohort analysis of a randomised, multicentre, diagnostic screening trial. Lancet Oncol 2016;17:590-9.

60. Yousaf-Khan U, van der Aalst C, de Jong PA, et al. Final screening round of the NELSON lung cancer screening 
trial: the effect of a 2.5-year screening interval. Thorax 2017;72:48-56.

61. Schreuder A, Schaefer-Prokop CM, Scholten ET, et al. Lung cancer risk to personalise annual and biennial follow-up computed tomography screening. Thorax 2018;73:626-33.

62. Pastorino U, Sverzellati N, Sestini S, et al. Ten-year results of the Multicentric Italian Lung Detection trial demonstrate the safety and efficacy of biennial lung cancer screening. Eur J Cancer 2019;118:142-8.

63. Robbins HA, Berg CD, Cheung LC, et al. Identification of Candidates for Longer Lung Cancer Screening Intervals Following a Negative Low-Dose Computed Tomography Result. J Natl Cancer Inst 2019;111:996-9.

64. Ganeshan D, Duong PT, Probyn L, et al. Structured Reporting in Radiology. Acad Radiol 2018;25:66-73.

65. Carter BW, Lichtenberger JP, Wu CC, et al. Screening for Lung Cancer: Lexicon for Communicating With Health Care Providers. AJR Am J Roentgenol 2018;210:473-9.

66. Bosmans JM, Peremans L, De Schepper AM, et al. How do referring clinicians want radiologists to report? Suggestions from the COVER survey. Insights Imaging 2011;2:577-84.

67. Vitzthum von Eckstaedt H 5th, Kitts AB, Swanson C, et al. Patient-centered Radiology Reporting for Lung Cancer Screening. J Thorac Imaging 2020;35:85-90.

68. Ravenel JG, Nance JW. Coronary artery calcification in lung cancer screening. Transl Lung Cancer Res 2018;7:361-7.

69. Takx RA, Išgum I, Willemink MJ, et al. Quantification of coronary artery calcium in nongated CT to predict cardiovascular events in male lung cancer screening participants: results of the NELSON study. J Cardiovasc Comput Tomogr 2015;9:50-7.

70. Watts JR, Sonavane SK, Snell-Bergeon J, et al. Visual scoring of coronary artery calcification in lung cancer screening computed tomography: association with allcause and cardiovascular mortality risk. Coron Artery Dis 2015;26:157-62.

71. Carr LL, Jacobson S, Lynch DA, et al. Features of COPD as Predictors of Lung Cancer. Chest 2018;153:1326-35.

72. Heuvelmans MA, Vonder M, Rook M, et al. Screening for Early Lung Cancer, Chronic Obstructive Pulmonary Disease, and Cardiovascular Disease (the Big-3) Using Low-dose Chest Computed Tomography. J Thorac Imaging 2019;34:160-9.

73. Chen A, Karwoski RA, Gierada DS, et al. Quantitative CT Analysis of Diffuse Lung Disease. Radiographics
2020;40:28-43.

74. Dirrichs T, Penzkofer T, Reinartz SD, et al. Extracoronary Thoracic and Coronary Artery Calcifications on Chest CT for Lung Cancer Screening Association with Established Cardiovascular Risk Factors - The "CT-Risk" Trial. Acad Radiol 2015;22:880-9.

75. Kim YW, Kim JH, Yoon SH, et al. Vertebral bone attenuation on low-dose chest CT: quantitative volumetric analysis for bone fragility assessment. Osteoporos Int 2017;28:329-38.

76. Margolies L, Salvatore M, Eber C, et al. The general radiologist's role in breast cancer risk assessment: breast density measurement on chest CT. Clin Imaging 2015;39:979-82.

77. Chen X, Li K, Yip R, et al. Hepatic steatosis in participants in a program of low-dose CT screening for lung cancer. Eur J Radiol 2017;94:174-9.

78. Silva M, Sverzellati N, Colombi D, et al. Pleural plaques in lung cancer screening by low-dose computed tomography: prevalence, association with lung cancer and mortality. BMC Pulm Med 2017;17:155.

79. Lee C. Screening for Lung Cancer: Effective Recruitment Methods. AJR Am J Roentgenol 2018;210:514-7.

80. Glazer GM, Ruiz-Wibbelsmann JA. The Invisible Radiologist. Radiology 2011;258:18-22.

81. Rosenkrantz AB, Pysarenko K. The Patient Experience in Radiology: Observations From Over 3,500 Patient Feedback Reports in a Single Institution. J Am Coll Radiol 2016;13:1371-7.

82. Rosenkrantz AB, Mason D, Kunzler NM, Lee J. The Radiologist as Direct Public Educator: Impact of Sessions Demystifying Select Cancer Screening Imaging Examinations. J Am Coll Radiol 2016;13:R38-42.

83. Tanner NT, Silvestri GA. Shared Decision-making and Lung Cancer Screening: Let's Get the Conversation Started. Chest 2019;155:21-4.

84. Green DB, Pua BB, Crawford CB, et al. Screening for Lung Cancer: Communicating with Patients. AJR Am J Roentgenol 2018;210:497-502.

85. Ciompi F, Chung K, van Riel SJ, et al. Towards automatic pulmonary nodule management in lung cancer screening with deep learning. Sci Rep 2017;7:46479. Erratum in: Sci Rep. 2017 Sep 7;7:46878.

86. Kadir T, Gleeson F. Lung cancer prediction using machine learning and advanced imaging techniques. Transl Lung Cancer Res 2018;7:304-12.

87. Field JK, deKoning H, Oudkerk M, et al. Implementation of lung cancer screening in Europe: challenges and 
potential solutions: summary of a multidisciplinary roundtable discussion. ESMO Open 2019;4:e000577.

88. Wang Y, Midthun DE, Wampfler JA, et al. Trends in the Proportion of Patients with Lung Cancer Meeting Screening Criteria. JAMA 2015;313:853-5.

89. Yang P, Wang Y, Wampfler JA, et al. Trends in

Cite this article as: Snoeckx A, Franck C, Silva M, Prokop M, Schaefer-Prokop C, Revel MP. The radiologist's role in lung cancer screening. Transl Lung Cancer Res 2021;10(5):23562367. doi: $10.21037 /$ tlcr-20-924
Subpopulations at High Risk for Lung Cancer. J Thorac Oncol 2016;11:194-202.

90. Mulshine JL, Avila RS, Conley E, et al. The International Association for the Study of Lung Cancer Early Lung Imaging Confederation. JCO Clin Cancer Inform 2020;4:89-99. 